\title{
Stereotactic body radiation therapy for the treatment of a post-chemotherapy remnant lung mass in extensive-stage small-cell lung cancer: A case report
}

\author{
SEUNG-GU YEO ${ }^{1}$ and MIN-JEONG KIM ${ }^{2}$ \\ ${ }^{1}$ Department of Radiation Oncology, Soonchunhyang University College of Medicine, Soonchunhyang University Hospital, \\ Cheonan, Chungnam 31151; ${ }^{2}$ Department of Radiology, Hallym University College of Medicine, \\ Hallym University Sacred Heart Hospital, Anyang, Gyeonggi 14068, Republic of Korea
}

Received March 26, 2015; Accepted April 22, 2016

DOI: $10.3892 /$ etm.2016.3359

\begin{abstract}
Stereotactic body radiation therapy (SBRT) can significantly improve the treatment outcomes of patients with inoperable stage I non-small-cell lung cancer. Similarly, a few case studies have reported the effectiveness of SBRT for stage I small-cell lung cancer (SCLC). However, no study has investigated the use of SBRT for extensive-stage SCLC (ES-SCLC). Compared with conventional RT, SBRT is able to deliver a higher radiation dose precisely and safely to small targets in short-duration treatments. The present study reports the outcome of a patient with ES-SCLC who responded favorably to initial chemotherapy and received SBRT for a residual mass in the peripheral lung. A 62-year-old female presented with pathologically determined SCLC at clinical stage T4N3M0-T4 as separate tumor nodules were present in different ipsilateral lobes. The patient received 6 cycles of standard chemotherapy with cisplatin and etoposide. The response of the patient to chemotherapy was evaluated using contrast-enhanced chest computed tomography and ${ }^{18} \mathrm{~F}$-fluorodeoxyglucose positron emission tomography-computed tomography. The only suspected residual viable tumor was a $1.5-\mathrm{cm}$ mass in the right upper lobe. Targeting this mass, intensity-modulated SBRT was performed with $48 \mathrm{~Gy}$ in 4 fractions and $6 \mathrm{MV}$ photons. In addition, prophylactic cranial irradiation was conducted with $25 \mathrm{~Gy}$ in 10 fractions. The patient is alive with no evidence of disease 4 years after treatment. SBRT toxicity was limited to radiation pneumonitis or fibrosis without pulmonary symptoms. This case report suggests the efficacy of SBRT in select
\end{abstract}

Correspondence to: Dr Min-Jeong Kim, Department of Radiology, Hallym University College of Medicine, Hallym University Sacred Heart Hospital, 22 Kwanpyeong-ro 170, Anyang, Gyeonggi 14068, Republic of Korea

E-mail: drkmj@hallym.or.kr

Key words: stereotactic body radiotherapy, chemotherapy, small-cell lung cancer, extensive stage
ES-SCLC patients with small residual lung disease following chemotherapy.

\section{Introduction}

Small-cell lung cancer (SCLC) accounts for $\sim 15 \%$ of all newly diagnosed lung cancers (1). SCLC is characterized by a rapid doubling time, high growth fraction, and the early development of distant metastases; $2 / 3$ of patients with SCLC present with extensive-stage disease (1). Systemic chemotherapy is the main treatment for all SCLCs, limited or extensive (1).

For limited-stage SCLC (LS-SCLC), the addition of thoracic radiation therapy (RT) to chemotherapy improves local control and survival, and concurrent chemoradiotherapy is considered the standard of care (2). For extensive-stage (ES)-SCLC, the purpose of RT is generally limited to palliation $(2,3)$. However, a recent multicenter randomized trial reported that thoracic RT lowered disease progression and improved survival rates in select patients with ES-SCLC who responded to initial chemotherapy (4).

Stereotactic body RT (SBRT) uses high-precision external beam RT to target small, well-defined tumors with a high radiation dose per fraction and short treatment courses (5). A high target dose is possible in SBRT without increasing normal tissue toxicity due to the steep dose gradients outside the target (6). Compared with conventional RT, SBRT substantially improved local control and survival in patients with inoperable early-stage non-SCLC (NSCLC) $(5,6)$. However, the benefits of SBRT have rarely been examined in SCLC.

The present study reports the long-term outcome of a patient with ES-SCLC who responded remarkably to chemotherapy and subsequently received thoracic SBRT for the post-chemotherapy control of remnant cancer in the lung periphery.

\section{Case report}

A 62-year-old female was referred to the Soonchunhyang University Cheonan Hospital (Cheonan, Korea) following cancer diagnosis and the completion of 6 cycles of standard chemotherapy (cisplatin and etoposide) from January to 

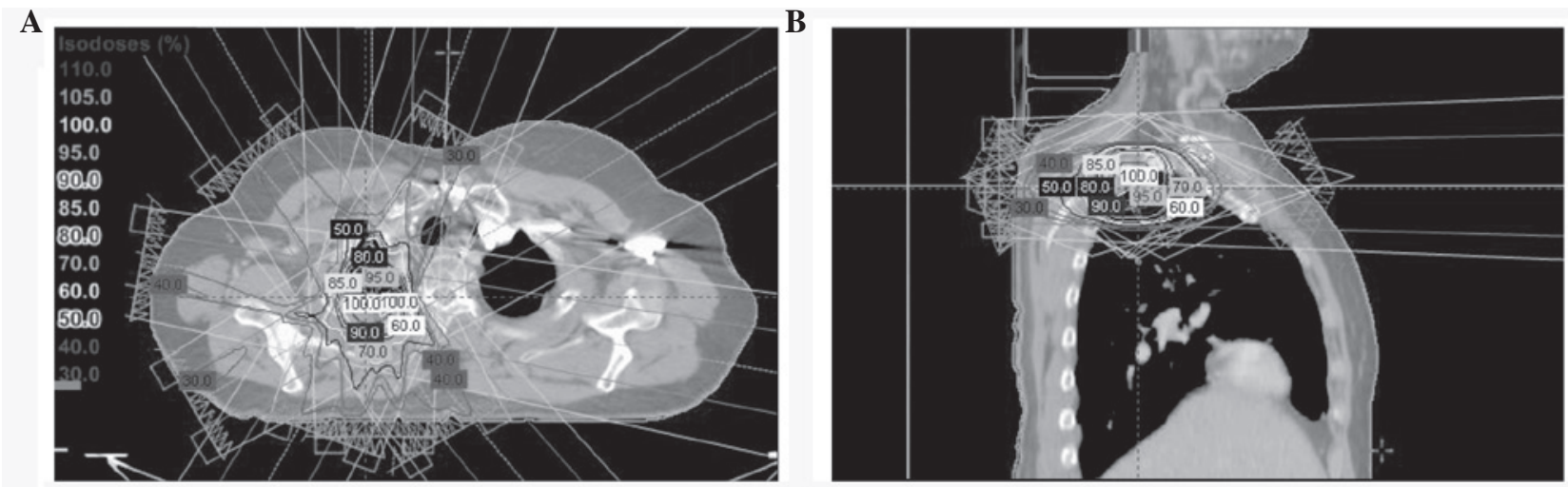

C

D
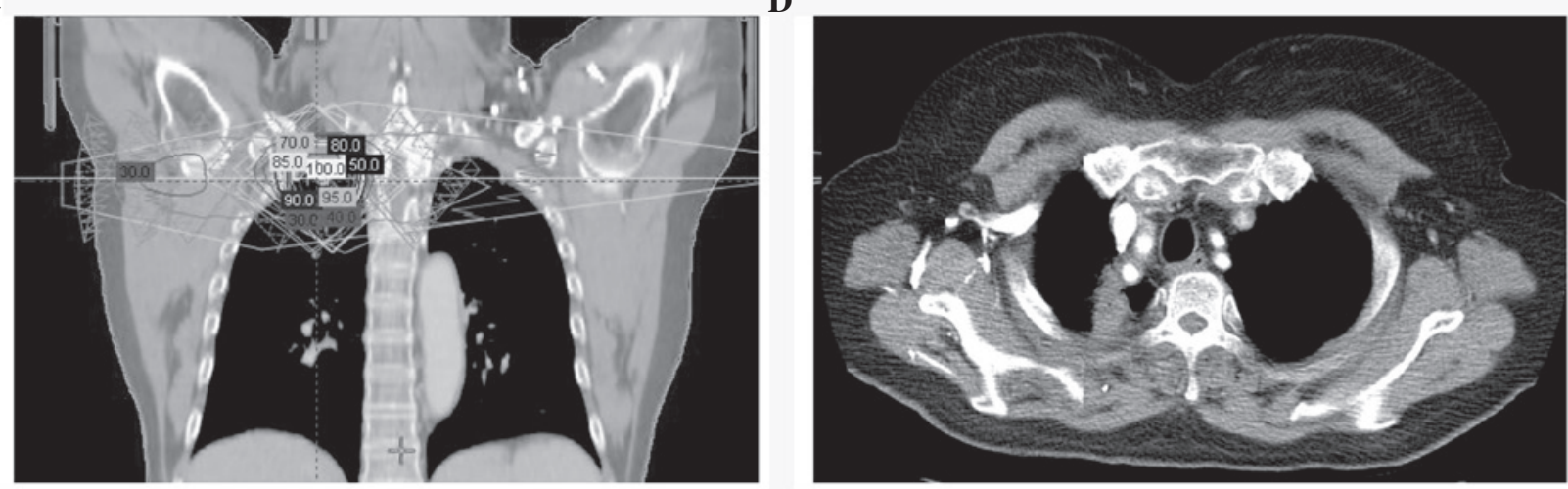

Figure 1. (A-C) Intensity-modulated SBRT plan using 7 non-opposing coplanar beams. The dose distribution with isodose lines $\geq 30 \%$ of the prescription dose is also shown. (D) Chest CT 40 months after SBRT shows focal non-enhanced consolidation in the right upper lobe, without ${ }^{18} \mathrm{~F}$-fluorodeoxyglucose uptake on the positron emission tomography-CT (not shown), indicating radiation-induced fibrosis. SBRT, stereotactic body radiation therapy; CT, computed tomography.

June 2011 at another hospital. The patient had pathologically determined SCLC, and the TNM stage was determined by a physical examination, pulmonary function tests, chest contrast-enhanced computed tomography (CT), ${ }^{18} \mathrm{~F}$-fluorodeoxyglucose positron emission tomography (PET)-CT and brain magnetic resonance imaging, which were performed prior to the patient's admission to our hospital. The clinical stage was determined to be extensive or T4N3M0 (IIIB), according to the American Joint Committee on Cancer staging system, 7th ed (7). The patient had separate tumor nodules in the right upper and middle lobes, and multiple metastatic lymph nodes were present in the mediastinum bilaterally.

The imaging studies were repeated in August 2011 to evaluate the response to chemotherapy, and a right upper lobe mass was thought to be a tumor remnant; the mass had a maximum diameter of $1.5 \mathrm{~cm}$ and a maximized standard uptake value of 3.5. After noting an excellent response to chemotherapy with a small remaining viable peripheral lung mass, SBRT was used at our hospital to treat the residual tumor of the patient.

SBRT was performed in August 2011 using a Novalis Tx system (Varian Medical Systems, Palo Alto, CA, USA; Brainlab, Feldkirchen, Germany). During the simulation process, the patient was immobilized in the supine position with her arms above her head. The simulation was conducted using 4-dimensional CT (Brilliance CT Big Bore; Philips Medical Systems, Cleveland, OH, USA) and a real-time position-management system (Varian Medical Systems). The gross tumor volume was delineated on each respiratory phase CT image using the 'lung window' setting. The clinical target volume was equivalent to the gross tumor volume. The internal target volume was created from the sum of the clinical target volumes during the 10 respiratory phases. The planning target volume was generated by adding a $0.5-\mathrm{cm}$ isotropic set-up margin around the internal target volume. Adjacent organs at risk were outlined, including the lungs, spinal cord, esophagus, trachea, proximal bronchial tree, heart, great vessels, ribs, and skin. The SBRT plan was created using the Eclipse treatment planning system (Varian Medical Systems) and $6 \mathrm{MV}$ photons, with inhomogeneity corrections. The fixed-field intensity modulated RT used 7 non-opposing coplanar beams (Fig. 1A-C). The dose fractionation scheme was $48 \mathrm{~Gy}$ in 4 fractions delivered on consecutive days, and the prescribed dose covered $95 \%$ of the planning target volume conformally. Prior to each treatment, cone beam CT was performed to minimize the set-up error. Prophylactic cranial irradiation (PCI) (8) was given in September 2011, as 25 Gy in 10 fractions using $6 \mathrm{MV}$ photons and two opposed lateral fields.

The patient was followed-up every 3 months for the first 2 years and every 6 months thereafter. A physical examination and chest $\mathrm{CT}$ were performed at each visit and PET-CT yearly. A complete response to SBRT was observed along with signs of radiation pneumonitis or fibrosis on follow-up imaging (Fig. 1D), although the patient developed no pulmonary symptoms. The patient is alive with no evidence of disease 4 years after treatment. The present study was performed in accordance with the guidelines of the Institutional Review Board and informed consent was obtained from the patient prior to commencing SBRT. 


\section{Discussion}

Currently, the use of thoracic SBRT in primary lung cancer is restricted to NSCLC (9). For patients with stage I NSCLC who are medically inoperable or refuse surgery, conventional RT has disappointing outcomes; the 3-year survival rate is $20-35 \%$, with local control rates of $30-40 \%$ (9). Since SBRT replaced conventional RT for these patients, there has been a marked improvement in patient prognosis; the 3-year survival rate is $\sim 60 \%$ and the local control exceeds $85-90 \%$, comparable to the outcomes after surgery $(9,10)$. The primary reason for poor tumor control with conventional RT is the insufficient target dose, which is $\sim 60$ Gy (11). Conversely, the minimum biological effective dose (BED) delivered by SBRT $>100 \mathrm{~Gy}_{10}$ (11).

Compared with NSCLC, few studies have investigated SBRT in SCLC (12-14). One reason is that $5 \%$ of SCLCs are stage I (15). Surgery can be considered instead of RT for stage I SCLC (16). For inoperable stage I SCLC, a few case studies have addressed the use of SBRT. Shioyama et al (12) reported the SBRT outcomes of 8 cases with stage I SCLC: 6 patients were inoperable and 2 refused surgery. The SBRT treatment plan was 48 Gy delivered in 4 fractions. Chemotherapy was given to 6 patients, and no PCI was performed. The 3-year local control and survival rates were $100 \%$ and $72 \%$, respectively. SBRT-associated toxicity of grade 2 or more was not observed (12). Videtic et al (13) reported 6 cases with inoperable stage I SCLC whose primary tumors were managed with SBRT. The SBRT treatment plan was 60 Gy in 3 fractions, $50 \mathrm{~Gy}$ in 5 fractions, or $30 \mathrm{~Gy}$ in 1 fraction. A total of 4 patients received chemotherapy and PCI. The 1-year local control and survival rates were $100 \%$ and $63 \%$, respectively. Only 1 case of grade 2 toxicity was observed, with no grade $\geq 3$ toxicity (13). Ly et al (14) reported the SBRT outcomes of 8 patients with inoperable stage I SCLC and 3 patients who received SBRT as a salvage treatment for recurrent stage I SCLC. The investigators suggested that SBRT is a reasonable approach for inoperable stage I SCLC, and stressed the importance of post-SBRT chemotherapy (14). These studies demonstrated the potential value of SBRT for SCLC, which was concordant with its recognized value in NSCLC. However, to the best of our knowledge, to date no study has explored curative effect of thoracic SBRT for the treatment of ES-SCLC.

Thoracic RT is not an established treatment for ES-SCLC, except for symptom palliation (2). However, a small number of randomized trials demonstrated the beneficial effects of conventional thoracic RT in select ES-SCLC patients $(4,17)$. Jeremic et al (17) randomized 109 ES-SCLC patients who showed both a complete response at distant lesions and at least a partial response at local lesions following 3 initial chemotherapy cycles. The group that also received thoracic RT (54 Gy/36 fractions, b.i.d.) showed better local control and survival compared with the control group receiving further chemotherapy alone (17). Recently, the results of a multi-institutional randomized controlled trial were reported (4). Slotman et al (4) analyzed the outcomes of 495 patients with ES-SCLC who responded to 4-6 initial cycles of chemotherapy. A group of 247 patients randomized to receive thoracic RT (30 Gy/10 fractions) and PCI showed improvement in terms of disease progression and survival compared with the control group, who received PCI and no thoracic RT (2-year survival, $13 \%$ vs. 3\%; $\mathrm{P}=0.004$ ) (4). There was a $50 \%$ reduction in intrathoracic recurrence when thoracic RT was added, although $>40 \%$ of the patients treated in this manner still had intrathoracic recurrence (4). The authors suggested that a higher radiation dose using advanced delivery techniques would be more efficacious (4). In the present study, SBRT with novel RT technology was used to deliver a high radiation dose precisely and safely. The BED of $30 \mathrm{~Gy} / 10$ fractions was $39.0 \mathrm{~Gy}_{10}$, whereas that of $48 \mathrm{~Gy} / 4$ fractions (the current case) was $105.6 \mathrm{~Gy}_{10}$.

The definition of the appropriate RT target volume for SCLC has not been fully defined. Regarding the RT target volume in LS-SCLC, recent studies have recommended that the post-induction chemotherapy tumor extent be considered and that elective nodal irradiation is not necessary (18-21). For ES-SCLC, the RT target volume in two randomized trials included pre-chemotherapy metastatic mediastinal or hilar lymph nodes $(4,17)$. Conversely, a phase II trial investigating thoracic RT for ES-SCLC used an RT target volume limited to the post-chemotherapy residual tumor (22). SBRT was selected for the present patient as only the right upper lobe mass was suspected to be residual tumor. Lung SBRT typically targets small masses located in the peripheral lung $(6,11)$. Chemotherapy response was evaluated using both chest $\mathrm{CT}$ and PET-CT. van Loon et al (23) compared ${ }^{18} \mathrm{~F}$-fluorodeoxyglucose PET- and CT-based selective nodal irradiation in SCLC and showed that the former yielded lower rates of isolated nodal failure and RT-associated toxicities. The results of the study suggested that PET-CT was the most reliable method for staging the mediastinum in SCLC, excluding pathological verification. This post-chemotherapy assessment was deemed to have been accurate since there has been no disease recurrence inside or outside the SBRT target volume.

In conclusion, a careful evaluation of the chemotherapy response is mandatory for the implementation of SBRT in ES-SCLC, of which the target volume is typically limited. When this condition is met, SBRT will enable enhanced local tumor control and patient survival compared with conventional RT encompassing a wide target volume or further chemotherapy without any RT. The results of the present case report suggests the efficacy of SBRT in select ES-SCLC patients who have a small amount of residual tumor in the peripheral lung following chemotherapy.

\section{Acknowledgements}

The present study was supported by the Soonchunhyang University Research Fund.

\section{References}

1. Hann CL and Rudin CM: Management of small-cell lung cancer: Incremental changes but hope for the future. Oncology (Williston Park) 22: 1486-1492, 2008.

2. Kong FM, Lally BE, Chang JY, Chetty IJ, Decker RH, Ginsburg ME, Kestin LL, Langer CJ, Movsas B, Videtic GM, et al: ACR appropriateness criteria ${ }^{\circledR}$ radiation therapy for small-cell lung cancer. Am J Clin Oncol 36: 206-213, 2013.

3. Pignon JP, Arriagada R, Ihde DC, Johnson DH, Perry MC, Souhami RL, Brodin O, Joss RA, Kies MS, Lebeau B, et al: A meta-analysis of thoracic radiotherapy for small-cell lung cancer. N Engl J Med 327: 1618-1624, 1992. 
4. Slotman BJ, van Tinteren H, Praag JO, Knegjens JL, El Sharouni SY, Hatton M, Keijser A, Faivre-Finn C and Senan S: Use of thoracic radiotherapy for extensive stage small-cell lung cancer: A phase 3 randomised controlled trial. Lancet 385: 36-42, 2015.

5. Soldà F, Lodge M, Ashley S, Whitington A, Goldstraw P and Brada M: Stereotactic radiotherapy (SABR) for the treatment of primary non-small cell lung cancer; systematic review and comparison with a surgical cohort. Radiother Oncol 109: 1-7, 2013.

6. Guckenberger M, Andratschke N, Alheit H, Holy R, Moustakis C, Nestle U and Sauer O; Deutschen Gesellschaft für Radioonkologie (DEGRO): Definition of stereotactic body radiotherapy: Principles and practice for the treatment of stage I non-small cell lung cancer. Strahlenther Onkol 190: 26-33, 2014

7. Edge S, Byrd DR, Compton CC, Fritz AG, Greene FL and Trotti A, (eds): AJCC Cancer Staging Manual. 7th edition. Springer, New York, NY, 2010.

8. Slotman B, Faivre-Finn C, Kramer G, Rankin E, Snee M, Hatton M, Postmus P, Collette L, Musat E and Senan S; EORTC Radiation Oncology Group and Lung Cancer Group: Prophylactic cranial irradiation in extensive small-cell lung cancer. N Engl J Med 357: 664-672, 2007.

9. Timmerman R, Paulus R, Galvin J, Michalski J, Straube W, Bradley J, Fakiris A, Bezjak A, Videtic G, Johnstone D, et al: Stereotactic body radiation therapy for inoperable early stage lung cancer. JAMA 303: 1070-1076, 2010.

10. Yeo SG and Kim ES: Efficient approach for determining four-dimensional computed tomography-based internal target volume in stereotactic radiotherapy of lung cancer. Radiat Oncol J 31: 247-251, 2013.

11. Kim MJ, Yeo SG, Kim ES, Min CK and Se An P Intensity-modulated stereotactic body radiotherapy for stage I non-small cell lung cancer. Oncol Lett 5: 840-844, 2013.

12. Shioyama Y, Nakamura K, Sasaki T, Ohga S, Yoshitake T, Nonoshita T, Asai K, Terashima K, Matsumoto K, Hirata H and Honda H: Clinical results of stereotactic body radiotherapy for Stage I small-cell lung cancer: A single institutional experience. J Radiat Res 54: 108-112, 2013.

13. Videtic GM, Stephans KL, Woody NM, Pennell NA, Shapiro M, Reddy CA and Djemil T: Stereotactic body radiation therapy-based treatment model for stage I medically inoperable small cell lung cancer. Pract Radiat Oncol 3: 301-306, 2013.
14. Ly NB, Allen PK and Lin SH: Stereotactic body radiation therapy for stage I small cell lung cancer: A single institutional case series and review of the literature. J Radiat Oncol 3: 285-291, 2014.

15. Ignatius Ou SH and Zell JA: The applicability of the proposed IASLC staging revisions to small cell lung cancer (SCLC) with comparison to the current UICC 6th TNM edition. J Thorac Oncol 4: 300-310, 2009.

16. de Hoyos A and DeCamp MM: Surgery for small cell lung cancer. Thorac Surg Clin 24: 399-409, 2014.

17. Jeremic B, Shibamoto Y, Nikolic N, Milicic B, Milisavljevic S, Dagovic A, Aleksandrovic J and Radosavljevic-Asic G: Role of radiation therapy in the combined-modality treatment of patients with extensive disease small-cell lung cancer: A randomized study. J Clin Oncol 17: 2092-2099, 1999.

18. Hu X, Bao Y, Zhang L, Guo Y, Chen YY, Li KX, Wang WH, Liu $\mathrm{Y}, \mathrm{He} \mathrm{H}$ and Chen $\mathrm{M}$ : Omitting elective nodal irradiation and irradiating postinduction versus preinduction chemotherapy tumor extent for limited-stage small cell lung cancer: Interim analysis of a prospective randomized noninferiority trial. Cancer 118: 278-287, 2012.

19. Colaco R, Sheikh H, Lorigan P, Blackhall F, Hulse P, Califano R, Ashcroft L, Taylor P, Thatcher N and Faivre-Finn C: Omitting elective nodal irradiation during thoracic irradiation in limited-stage small cell lung cancer - evidence from a phase II trial. Lung Cancer 76: 72-77, 2012.

20. Ricardi U, Badellino S and Filippi AR: Stereotactic radiotherapy for early stage non-small cell lung cancer. Radiat Oncol J 33: $57-65,2015$

21. Lee J, Lee J, Choi J, Kim JW, Cho J and Lee CG: Early treatment volume reduction rate as a prognostic factor in patients treated with chemoradiotherapy for limited stage small cell lung cancer. Radiat Oncol J 33: 117-125, 2015.

22. Yee D, Butts C, Reiman A, Joy A, Smylie M, Fenton D, Chu Q, Hanson J and Roa W: Clinical trial of post-chemotherapy consolidation thoracic radiotherapy for extensive-stage small cell lung cancer. Radiother Oncol 102: 234-238, 2012.

23. van Loon J, De Ruysscher D, Wanders R, Boersma L, Simons J, Oellers M, Dingemans AM, Hochstenbag M, Bootsma G, Geraedts W, et al: Selective nodal irradiation on basis of (18) FDG-PET scans in limited-disease small-cell lung cancer: A prospective study. Int J Radiat Oncol Biol Phys 77: 329-336, 2010 . 